\title{
Mechanical and Tribological Properties of Nanoparticles Aggregates determined using in situ AFM in the TEM
}

\author{
Audrey Molza ${ }^{1}$, Jean-Louis Mansot ${ }^{1,2}$ Maxime J-F Guinel $^{3}$ and Laurent Legras ${ }^{4}$ \\ ${ }^{1}$ Groupe de Technologie des Surfaces et Interfaces (GTSI EA 2432), \\ ${ }^{2}$ Centre Commun de Caractérisation des Matériaux des Antilles et de la Guyane $\left(\mathrm{C}^{3} \mathrm{MAG}\right)$, Université \\ des Antilles et de la Guyane, Faculté des Sciences Exactes et Naturelles, BP 250, 97157 Pointe à Pitre \\ Cédex, France \\ ${ }^{3}$ Departments of Chemistry and Physics, College of Natural Sciences, University of Puerto Rico, PO \\ Box 70377, San Juan, Puerto Rico 00936-8377, U.S.A.
}

${ }^{4}$ EDF - R\&D, Departements MMC et MAI, Avenue des Renardières, Les Renardières, 77818 Moret sur Loing Cedex, France

The tribological properties of nanoparticles used as solid lubricants were recently shown [1] to not only depend on their morphology, structure and chemical composition but also on their individual behavior -at the nanoscale - in the sliding interface. In this presentation, we will show the results of in situ experiments performed in the transmission electron microscope (TEM) using a specimen holder fitted with an atomic force microscope (AFM) cantilever. The samples used were small aggregates of carbon nanoparticles and partially fluorinated derivatives. Images were recorded and quantitative data extracted in order to determine their nano-mechanical and tribologic behavior when submitted to compressive and shear stresses.

The graphitized carbon black aggregates were purchased from Carbon Superior Graphite (Pureblack ${ }^{\circledR}$ 205-110). The fluorinated carbon blacks (fluor to carbon atomic ratio: 0.16) were prepared at the Institut de Chimie de Clermont-Ferrand (France). They were obtained by the direct fluorination in a Monel reactor under $\mathrm{F}_{2}$ atmosphere at $360^{\circ} \mathrm{C}$ for 3 hours. More details can be found elsewhere [2]. The materials were examined using a high resolution TEM (HRTEM, JEOL JEM-2200FS, operated at 200 $\mathrm{kV}$ and equipped with an in column Omega energy filter). The in situ experiments were carried out using a Nanofactory Instruments Inc. specimen holder.

The first series of experiments were concerned with the aggregate brittleness induced by the fluorination process. The aggregates were composed of individual spherical particles (diameters of about 10 to 70 $\mathrm{nm}$ ) with sizes in the range of several tens of nanometers to several micrometers. The fluorination process leads to the formation of an external fluorinated layer (less than $10 \mathrm{~nm}$ thick) and may increase the brittleness of the aggregates. They showed remarkable stability during the in situ nano-tribology experiments and the particles remained strongly bonded to each others. Individual aggregates behaved elastically when relatively large compressive stresses were applied. Figure 1 shows three TEM images recorded from a single aggregate before, while and after compression with the AFM tip. The aggregates undergo large deformations without significant damages and the deformation is elastic.

The second series of experiments were concerned with the quantitative measurement of the static friction coefficient between the AFM tip and the aggregates of pure graphitized carbon blacks. In that case, a thin silicon cantilever was soldered (using the focused ion beam) onto a support grid normal to the friction direction (a micrograph is shown in Figure 2). The carbon nanoparticles were deposited on the tip of this cantilever and were submitted to normal loads by the AFM tip, in situ. When lateral forces 
were applied, the cantilever elastically deformed and allowed for the measurement of the applied lateral force (the elastic deformation is calibrated). Distances measured from the images recorded in situ were used to determine the lateral force when the AFM tip slid onto the carbon nanoparticles suddenly (stick slip process). The ratio between this force and the normal load corresponds to the static friction coefficient.
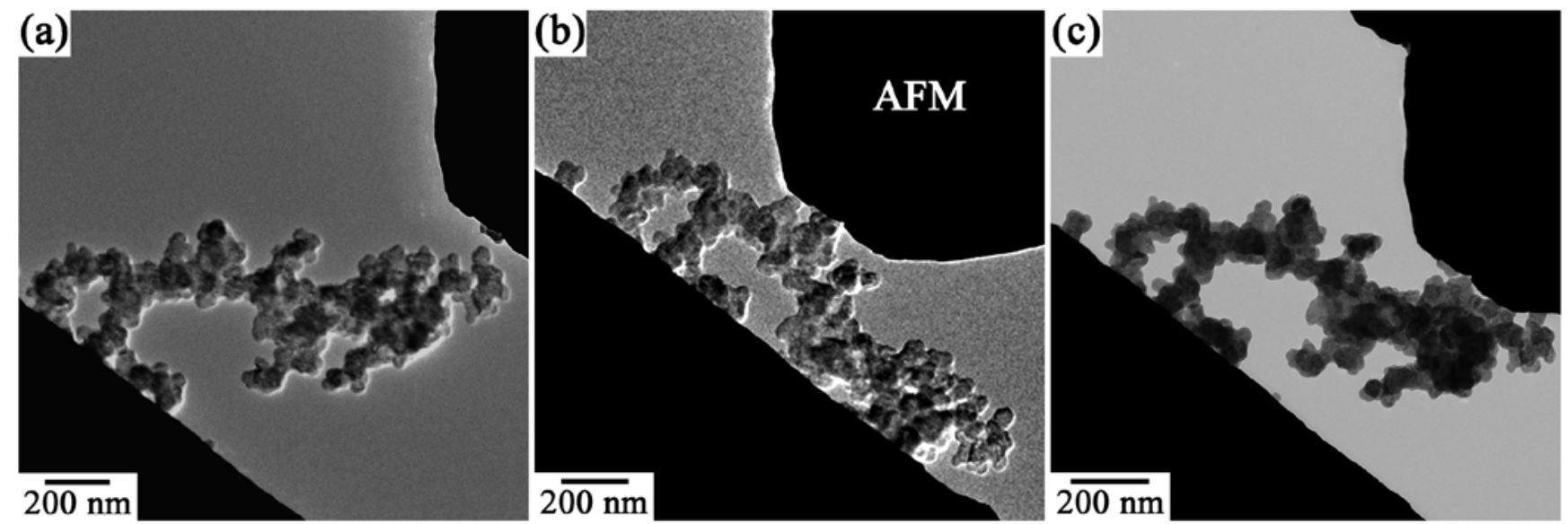

Figure 1. TEM images recorded from a single aggregate (a) before, (b) while and (c) after compression with the AFM tip.

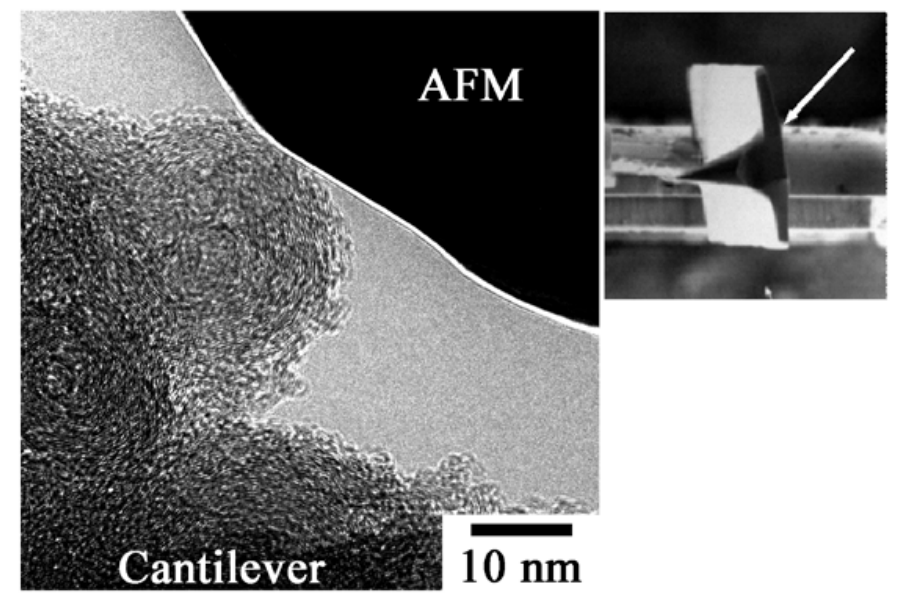

Figure 2. TEM image recorded in situ during the friction experiment showing few carbon black particles standing onto the cantilever and in contact with the AFM tip. The inset shows a micrograph of the cantilever attached onto a support grid.

\section{References}

[1] Thomas, P., Himmel, D., Mansot, J.L., Dubois, M., Guerin, K., Zhang, W. and Hamwi, A., Tribological properties of fluorinated carbon nanofibers. Tribo. Lett. (2009), vol. 34, pp. 49-59.

[2] Kannan A.M., Menghal A. and Barsukov, I.V., Gas diffusion layer using a new type of graphitized nano-carbon Pureblack ${ }^{\circledR}$ for proton exchange membrane fuel cells. Electrochemistry Communications (2006), vol. 8, pp. 887-91.

[3] The authors acknowledge the Institut de Chimie de Clermont-Ferrand for providing the fluorinated materials, the Ministère de la Recherche, the Conseil Régional de la Guadeloupe, the Conseil Régional de la Martinique, the Fonds Social Européen (FSE) and the Fonds Européens de Développement Régional (FEDER) for their financial supports. 